\title{
MODELOS FORMALES Y GENERATIVOS DE ENUNCIADOS NORMATIVOS
}

1. Es corriente en la literatura especializada definir, desde un punto morfológico, la noción de norma como un enunciado modal. Suele caracterizársela como un enunciado modal de deber-ser - ein Sollsatz-, entendiendo esa expresión como un verbo. Así Gioja, en una temprana interpretación del pensamiento kelseniano, formulada en el ensayo de 1949 "El tema de la Teoría Pura del Derecho", 1 apunta que "Kelsen comienza buscando una definición de la esencia del derecho; lo importante - continúa- consiste que en esa búsqueda se le muestra en un momento dado, una nueva estructura lógica: el juicio de 'deber ser', y que en vez de pasar a su lado sin atenderla, toda su preocupación apunta ahora al desentrañamiento y análisis de lo que constituye la forma de todo conocimiento normativo". Kelsen, como es sabido, estima que el conocimiento de las normas de un derecho positivo se formula en proposiciones jurídicas caracterizadas específicamente como Sollsätze, cuya función sería la de describir Sollnormen; la palabra Sollen -el verbo modal "deber" - sería un predicado modal utilizado teóricamente para enunciar los diversos modos que aparecen como sentidos de los actos jurídicamente regulados. ${ }^{2}$ Es una tesis sumamente frecuente en el neo-kantismo la afirmación de que los enunciados éticos de alguna suerte se distinguen por el recurso a la modalidad expresada por el Sollen, modalidad distintiva que, sean cuales fueren sus alcances epistemológicos o metafísicos, aparece también como una característica lingüística.

La definición de norma como enunciado modal de deber ser se encuentra también en pensadores ajenos a las preocupaciones teóricas de los juristas, como Husserl, 3 quien entiende los enunciados normativos como proposiciones dóxicas afectadas de una Sollensmodalität; y, naturalmente, en los escritores que se han ocupado de desarrollar la lógica propia de los enunciados normativos, como Georg von Wright ${ }^{4}$ y A. Ross Anderson, ${ }^{5}$ para limitarnos a la mención de dos de los iniciadores de esos estudios.

1 A. Gioja, El tema de la Teoria Pura del Derecho, Bs. Aires, 1949 (también publicado en La Ley, 24 de junio de 1949), p. 14. El pensamiento de Gioja sobre el punto naturalmente evolucionó al tomar contacto con las filosofias del lenguaje y las lógicas y semántica modernas.

2 H. Kelsen, Reine Rechtslehre ( $2^{\mathrm{a}}$ ed. 1966$), \S 4$, b y $\S 16$, especialmente pp. 17 y nota.

3 E. Husserl, Ideen zu einer reinen Phänomenologie und phänomenologische Philosophie I, $\$ 134$. Para los manuscritos sobre lógica normativa de 1920 , véase R. J. Vernengo, La ética de Husserl, Bs. Aires, 1968.

4 Cf. la última exposición extensa en Georg von Wright, An Essay in Deontic Logic and the General Theory of Action (Acta Philosophica Fennica, 1968).

5 A. Ross Anderson, "The Formal Analysis of Normative Systems" (1956), reproducido en The Logic of Decision and Action (compilador Nicholas Rescher, Pittsburgh, 1967). 
Claro está que esa caracterización, por suficiente que sea para un lenguaje artificial, no es excesivamente satisfactoria cuando pretendemos aplicarla a los enunciados normativos formulados en un lenguaje natural. La ciencia del derecho, por cierto, continúa recurriendo casi exclusivamente a los recursos expresivos de los lenguajes naturales y de ahí que no sean de extrañar los avatares que la definición misma de norma, o de enunciado normativo, sufre en Kelsen. La interpretación teórica de los enunciados normativos en los lenguajes naturales como enunciados de deber ser ha ocasionado dificultades de análisis, acaso insuperables, que obligan perentoriamente a continuar la tarea de mejorar los modelos propuestos. Entiendo que las contribuciones efectuadas por la filosofía analítica de los lenguajes naturales y las lógicas modales deónticas son ciertamente de gran importancia; algunas de las técnicas analíticas propuestas por las gramáticas transformacionales, sin embargo, pueden darnos, según creo, una herramienta más útil para aproximar los análisis efectuados sobre modelos construidos en lenguajes artificiales al plano de los lenguajes naturales utilizados efectivamente por las ciencias jurídicas. Este texto es un ensayo en esa dirección.

2. Partamos, como corresponde, de comprobaciones suficientemente notorias. Todo jurista aceptaría sin más que enunciados, como los siguientes

(2.1) Las palabras de los contratos y convenciones deben entenderse en el sentido que les da el uso general.

(2.2) Los contratos para los cuales se establecen determinadamente formas o solemnidades particulares, no producirán acción en juicio.

(2.3) La condición resolutoria se entiende implicitamente comprendida en todos los contratos bilaterales o sinalagmáticos, han de ser entendidos como normas o, como preferiremos decir, como enunciados normativos: afirman que cierta cosa o hecho es obligatorio, permitido, prohibido, etc. Se trata de textos que forman parte del derecho positivo argentino. De aceptarse que todos ellos, sea cual fuere su tenor y su apariencia gráfica, sean normas, cabría concluir que, en principio, no contamos en castellano con un signo lingüístico único, con un morfema o lexema característico, que sea portador exclusivo de la característica normativa que se atribuye a la totalidad de esos enunciados. Se trata de cosa aceptada: toda norma, se afirma, puede redactarse bajo la forma de un enunciado de deber ser; puede aparecer también como una oración puramente declarativa con el verbo principal en futuro e, inclusive, en ciertos contextos, no contar con ningún elemento léxico o sintáctico que la diferencie de una oración puramente enunciativa. En muchos lenguajes también vale la conversa: oraciones con el verbo auxiliar "deber" son utilizadas para formular una proposición enunciativa de futuro, sin que la aparición de ese verbo le otorgue siempre características normativas. ${ }^{6}$ Vale decir: no es cosa evidente cuál sea el componente lingüís-

6 Cf. O. Jespersen, The Philosophy of Grammar, edición 1968, p. 261 y 300; tam- 
tico -elemento léxico, componente morfológico o estructura sintáctica- que nos permite calificar a un enunciado como una norma. La remisión al contexto de uso parece, pues, la solución más recomendable. Ya Wittgenstein en 1930 señalaba que todo enunciado prescriptivo (o norma) puede ser entendido como un enunciado declarativo o descriptivo y viceversa: ${ }^{7}$ la diferenciación no se da en el puro plano del lenguaje, si se abstrae de la situación en que se lo utiliza. Podría pensarse que la definición propuesta arriba --norma como enunciado de deber ser- fuera inaplicable a los enunciados de sentido normativo que aparecen en los contextos normales de uso de los lenguajes naturales, limitándose a ser una construcción teórica destinada al análisis de ciertos lenguajes artificiales.

3. En consecuencia: la circunstancia de que un enunciado que efectivamente es entendido como normativo aparezca como una oración de deber ser, no es más que una contingencia estilística. La apariencia superficial, la forma externa, del enunciado no hace a su sentido normativo. En la terminología usual de las gramáticas generativas, diríamos que el "deber ser" es un componente contingente de la estructura superficial del enunciado normativo sometido a análisis. Y tratándose, por lo tanto, de cuestión que hace, no a la apariencia textual del enunciado, sino a su sentido, cabe suponer fundadamente que aquel elemento que dota a un enunciado de un sentido normativo, elemento que no hemos podido discernir siempre en las estructuras lingüisticas superficiales, integra la estructura profunda, léxica o sintáctica, de los enunciados considerados normativos; las transformaciones que permitirian el tránsito de la estructura profunda, determinante del sentido normativo percibido, al enunciado lingüístico que, gráfica o fonéticamente, expresa ese sentido modal, serian múltiples, toda vez que, según revelan los ejemplos (2.1)-(2.3) del parágrafo anterior, hasta es posible que en la estructura superficial no aparezca rasgo alguno portador de normatividad. En ese caso - nuestro ejemplo (2.3) - la transformación pertinente que produce la redacción superficial transcripta, manteniendo el sentido normativo existente en la estructura profunda, importa, entre otras operaciones, la supresión del rasgo que en esa estructura profunda traduce el específico sentido normativo.

4. Todo enunciado normativo, suponemos, cuenta con una característica o sentido modal típico, justamente el que lo hace entender, sea cual fuere su contenido, como norma. Entre una oración enunciativa cualquiera $\rightarrow$ sea

(4.1) El comprador paga el precio, $-\mathrm{y}$ el enunciado normativo asociado:

(4.2) El comprador debe pagar el precio,

biẻn: K. Döhmann, "Die sprachliche Darstellung der Modalfunktoren", $\$ 8.222$ ss. (en Logique et Analyse, no 13-14, 1961); A. Ross, Directives and Norms (1968), § 18, passim.

7 L. Wittgenstein, Philosophische Bemerkungen (edición 1964), II, §14, p. 59 . 
advertimos una diferencia, no en la referencia propiamente dicha, sino en su sentido modal, que. puede reflejarse o no - según vimos- en las respectivas estructuras superficiales. Vale también, según el dictamen premonitorio de Wittgenstein, que todo enunciado, aparezca modalmente neutro o modalizado de alguna otra suerte, puede ser interpretado como una norma, en la medida en que contemos con transformaciones que nos permitan reducirlo a alguna estructura profunda dotada del rasgo abstracto característico de los sentidos normativos.

Por de pronto, baste señalar que en los lenguajes naturales -como sucede en castellano- pareciera haber mecanismos operantes sobre los rasgos segmentales y el orden sintáctico de las estructuras profundas, que permiten construir a partir de una oración o frase enunciativa cualesquiera, los respectivos enunciados normativos asociados. Esos mecanismos, va de suyo, diferirán según sean los recursos expresivos del lenguaje en cuestión: dado que, superficialmente, hasta un giro imperativo puede traducir un sentido normativo profundo, habrá que admitir que hasta una variación suprasegmental es apta para expresar lingüísticamente una fórmula normativa. Con todo, refiriéndonos al par ordenado de transformación oración enunciativa/enunciado normativo, cabe formular ciertas precisiones que la lógica y la filosofía moral se han preocupado en destacar desde hace mucho tiempo.

5. Desde Hume, quien hizo hincapié en el problema, se suele apuntar que no se da implicación lógica, ni, por lo tanto, relación de deducibilidad factible, entre la oración enunciativa (simbolicémosla $p$ ) y los enunciados normativos asociados (pongamos: $O P$ y $P p$, que leemos "obligatorio $p$ " y "permitido $p$ "). Vale decir que no pueden admitirse inferencias como las siguientes:

(5.1) $p \rightarrow O p$ (donde la flecha simboliza la derivación);

(5.2) $O p \rightarrow p$

(5.3) $P p \rightarrow p$

(5.4) $p \rightarrow P p$, etcétera.

Esta restricción - conocida como postulado de Hume- es válida cuando $p$ es un enunciado contingente o fáctico, puesto que la restricción puede ser eliminada cuando $p$ sea el símbolo de una tautología. Es un axioma frecuente en los sistemas corrientes de lógica deóntica aceptar la derivación de un enunciado normativo, cuando el enunciado declarativo asociado es tautológico. Por ejemplo, a partir del principio de tercero excluido, podemos inferir una tautología deóntica asociada:

(5.5) CAPNPOApNp, que por distribución nos da AOpONp. Dado que aceptamos sin más que entre los enunciados normativos y los enunciados puramente declarativos contenidos en las fórmulas $(5 \cdot 1)-(5.4)$ no hay posibilidad de equivalencia, sinonimia o deducibilidad, tendremos que aceptar que la relación lingüística que permite, sin embargo, obtener unos de los otros, 
no es una relación semántica entre las denotaciones, ni una relación lógica entre los valores de verdad que cada expresión exhiba.

De manera que, respetando las restricciones lógicas resultantes del postulado de Hume, debemos suponer una función gramatical transformacional que permita obtener, a partir de una oración, la norma asociada, y viceversa. Designaremos esa operación transformacional gramatical con la letra $T$ antepuesta a las variables proposicionales. En los lenguajes naturales, diremos, son válidas las transformaciones siguientes:

(5.6) $T p O p ; \mathrm{y}$,

(5.7) TOpp,

aun cuando su efectiva realización puede suscitar complicados problemas estilísticos. Nos interesa investigar la operación $T$ en la medida en que ello nos permita comprender el alcance de las interpretaciones tradicionales entre los juristas de los enunciados normativos como enunciados de deber ser.

6. La definición inicial -norma como enunciado de deber ser- puede ser vista como una propuesta de formulación paradigmática de una suerte de modelo lógico-lingüístico destinado al análisis de la estructura profunda o sentido de todas aquellas expresiones superficiales que son entendidas efectivamente como normas. Como indicaba Gioja, en el texto mencionado en el $\S 1$, el juicio de deber ser, como nueva estructura lógica, constituiría la forma de todo conocimiento normativo, y, a la vez, momento esencial de la definición del derecho mismo. Quizás interese, ante la importancia concedida a esa propuesta analítica, revisar algunas de las interpretaciones que la definición indicada ha merecido en la literatura, aunque no hayan echado mano de los recursos teóricos concedidos por las gramáticas generativas. Dos modelos de análisis, creo, son los más frecuentemente utilizados: el enunciado normativo es construido como una modalización específica de la oración afirmativa, o bien, el enunciado normativo es pensado como un enunciado de nivel lingüístico distinto del de su enunciado asociado. ${ }^{8}$

El primer modelo interpreta al verbo "deber ser" $\longrightarrow$ al rasgo superficial correspondiente- como una suerte de operador lógico modal, similar en muchos aspectos a los rasgos léxicos que modalizan bajo el modo de la necesidad a un enunciado declarativo. El verbo auxiliar modal germánico sollen desempeña esa función: el enunciado no modalizado se convierte en una Sollsatz. Kelsen, en la última versión de la Teoría Pura, ${ }^{9}$ se inclina decididamente por esta versión: el verbo sollen es expresión simbólica de las modalidades que adoptan los enunciados, cuando se convierten en enunciados normativos. Podemos aludir a este modelo analítico invocando el tí-

8 Para un planteo relacionado con este segundo modelo, véase el ensayo de C. Alchourón, "Logic of Norms and Logic of Normative Propositions", en Logique et Analyse, n? 47,1969 .

9 H. Kelsen, op. cit., $\S_{4}$ b), especialmente, p. 5-6. 
tulo de un temprano ensayo de R. Feys: "la expresión modal del deber ser".10

La regla de formación general utilizada en las lógicas normativas primeras de von Wright y de Ross Anderson reza: si a una expresión bien formada de la lógica de enunciados se le antepone un operador modal deóntico (como $O$ ) obtenemos un enunciado normativo: $O p$. Operadores de este tipo, han sido denominados, como sabemos, operadores modales deónticos, y los enunciados normativos resultantes de su aplicación, enunciados normativos o normas. En este respecto, los operadores deónticos se desempeñan en forma similar a los operadores modales clásicos: necesario, posible, etcétera. Desde el ensayo inicial de von Wright, "Deontic Logic" de 1951, el estudio formal de este tipo de enunciados normativos ha correspondido a una rama especial de la lógica modal: la de las lógicas deónticas.

El operador $O$-que utilizaremos como típico de la clase de los operadores deónticos - ofrece, sin embargo, ciertas peculiaridades que lo diferencian en algún respecto de los operadores modales clásicos. Veamos algunas de ellas: por de pronto, las reglas de formación que lo incluyen, destinadas a lograr enunciados normativos a partir de enunciados declarativos, difieren según sea el sistema lógico deóntico que se adopte, circunstancia que si bien no es objetable mientras nos movamos en el terreno de lenguajes artificiales construidos para fines específicos, es un tanto desconcertante en cuanto pueda pensarse que las reglas de formación propuestas sean modelos analíticos de las reglas de transformación $T$ de algún lenguaje natural. Así, por ejemplo, los sistemas propuestos difieren en cuanto a la viabilidad de fórmulas como las siguientes:

(6.1) $O C p O q$;

(6.2) $O O p$.

La primera exhibe un enunciado deóntico, $O q$, incluido en el dominio del modalizador deóntico del condicional; el segundo ejemplifica una oración cuya modalización deóntica ha sido reiterada. Nuestras intuiciones lógicas, de existir, no nos dan criterios explícitos y claros para decidirnos sobre la aceptabilidad de enunciados complejos análogos a los mencionados. De ahí, sin duda, que el propio von Wright cambiara de opinión al respecto, considerando en un principio que enunciados modales como los ejemplificados no constituyen fórmulas bien formadas aceptables en los sistemas propuestos. ${ }^{11} \mathrm{Y}$ en parte el rechazo de la propuesta de Ross Anderson, de reducir los enunciados deónticos a enunciados aléticos, propuesta vista como una versión de la falacia naturalista, proviene de que la constante $S$ (sanción) de alguna manera es interpretada como una proposición deóntica inmersa en un contexto modal alético.

10 R. Feys, "Expression modale du 'devoir être", en Journal of Symbolic Logic, ${ }_{195}^{6,}$ $\mathrm{n}^{0} 54$, p. $88 / 89$.

11 Cf. op. cit., en la nota 4, capítulo I, $\$ 3$. 
Inclusive un enunciado deóntico característicamente bien formado, como $O p$, por ejemplo, suele manifestar una extraña ambigüedad en sus posibles interpretaciones semánticas. En Kelsen, como es bien sabido, $O p$ podría ser considerado la fórmula simbólica de una norma propiamente dicha, como de un enunciado referente a una norma. ${ }^{12}$ Sucede que es posible pensar que los modalizadores deónticos, como $O$, no constituyen operadores lógicos en cuanto no expresan una función. El operador lógico típico, como la negación $N$, expresa una función cerrada, en cuanto tanto el dominio como el codominio de la función integran el mismo conjunto: el operador $N$ (negación) aplicado a un enunciado $p$, produce el enunciado $N p$, que también es un enunciado. Justamente ello es lo característico de los operadores que son funciones de verdad (truth-functional). El operador $O$ aplicado a un enunciado -esto es, al miembro de un conjunto, cuya nota definitoria es el contar con un valor de verdad determinado- resulta en un producto lingüístico que, como surge del postulado de Hume, rechaza esa característica. La función determinada por $O$ sería una función abierta, y el campo de la relación determinada por la regla de formación examinada, no constituye un producto cartesiano homogéneo. Puesto que ¿cuál es en realidad, lógicamente hablando, el campo de la relación definido por un operador $O$ que enlaza enunciados, o clases de enunciados, con normas o clases de normas? Estas últimas no son expresiones que satisfagan estrictamente la definición misma de enunciado que se adopta para definir la clase constituyente del dominio de la relación. Mientras que el dominio de la relación establecida por la regla de formación es una clase definible extensionalmente, el codominio de la relación sólo permite recurrir a una clase definida intencionalmente.

7. Sea ello como fuere $-y$ la literatura que examina los problemas derivados de las posibles interpretaciones de este primer modelo es abundante-, lo cierto es que la regla de formación que permite obtener un enunciado normativo, como $O p$, a partir de $p$, mediante la aplicación más o menos irrestricta del operador modal $O$, no puede ser vista normalmente como un paradigma que sirva para explicar la construcción de un enunciado normativo en un lenguaje natural, en cuanto el modelo nada aclara sobre cuáles sean los procesos de transformación léxica o sintáctica que deba sufrir una estructura profunda de sentido normativo para aparecer en el enunciado normativo que superficialmente entendemos como una norma. Por lo menos, no hay razón alguna para pensar que las reglas de formación, más o menos complejas, introducidas para construir los diversos lenguajes formales artificiales de que se ocupan los sistemas de lógicas deónticas, puedan ser consideradas sin más como reglas de formación o transformación de un lenguaje natural en el que aparecen enunciados como los mencionados en (2.1)-(2.3).

12 Cf. op. cit., especialmente $\S 16$. Véase también: A. Gioja, "La ciencia juridica positiva y el jusnaturalismo", en Revista Juridica de Buenos Aires, 1961, IV. 
La interpretación lingüistica más simple de la transformación utilizada cuando pasamos de una oración $p$ a la norma $O p$-de una oración enunciativa a la norma asociada-, transformación que constituiría el analogon de la regla de formación de las lógicas deónticas, sería la que postula una transformación en la estructura de la oración enunciativa, mediante la adición de un verbo auxiliar modal. El árbol generativo siguiente configuraría la estructura profunda de (4.1):

$(7.1)$

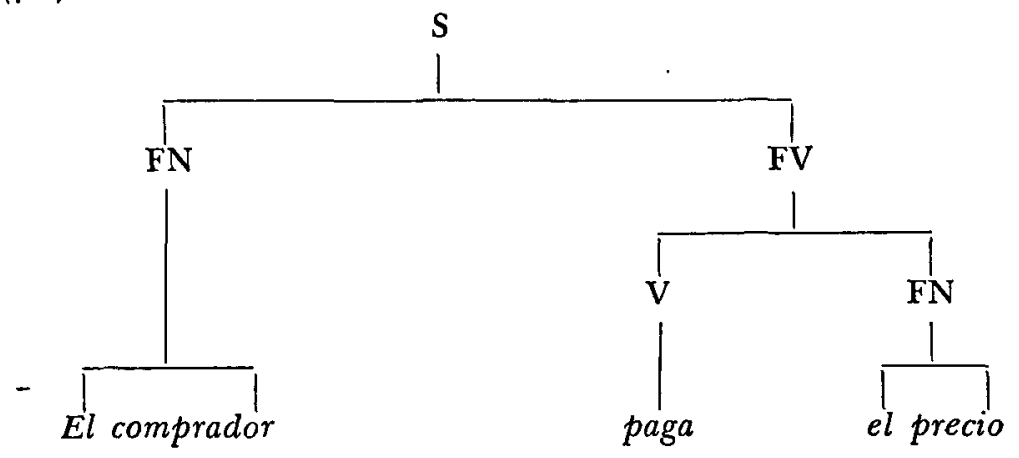

La oración normativa (4.2) tendría, en cambio, el siguiente aspecto:

$(7.2)$

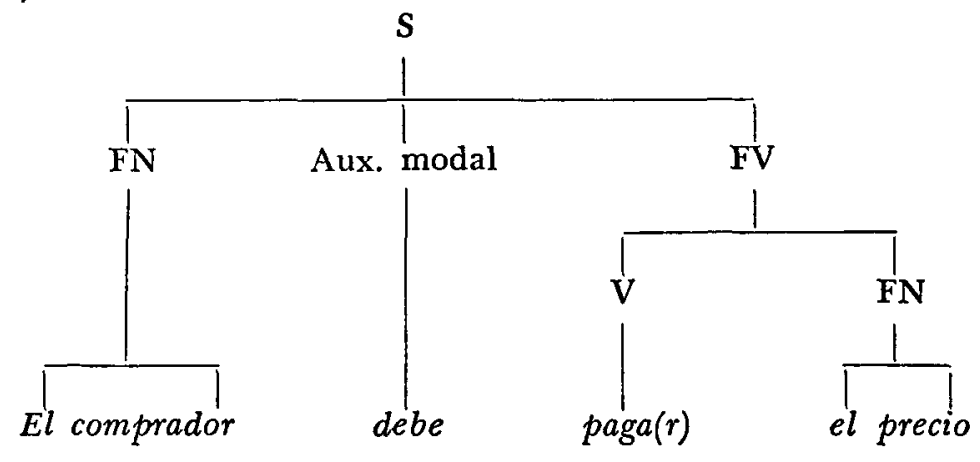

La introducción del auxiliar modal deóntico, como constituyente de la estructura profunda, acarrearía ciertas transformaciones obligatorias en la frase verbal. Asi, en castellano, por lo menos, el verbo principal ("paga") pierde sus rasgos distintivos (persona, tiempo, etc.), para adoptar la forma neutra del infinitivo; aquellos rasgos verbales distintivos son incorporados al auxiliar modal. 
Esta reconstrucción de la estructura profunda de un enunciado normativo no es satisfactoria. Podría pensarse que existe una transformación que genera, en la estructura profunda, un enunciado normativo a partir del enunciado declarativo, de suerte que el término modal ("debe") constituye en realidad un rasgo superficial resultante de la aplicación de esa transformación. (4.2) tendría que ser pensada de esta manera:

$(7 \cdot 3)$

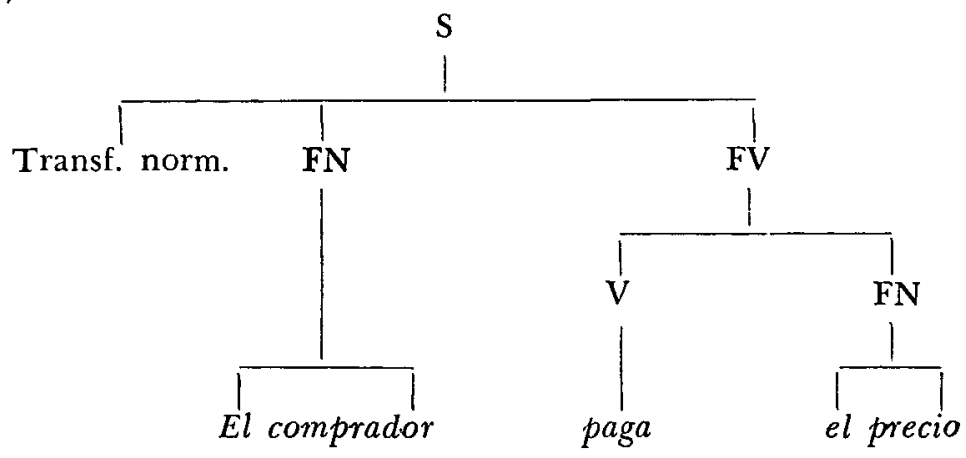

Sólo que no conocemos cuáles sean las reglas precisas, en castellano, por ejemplo, de la transformación normativa postulada en (7.3); y el constituyente introducido en el árbol de (7.2), como un verbo auxiliar modal, no acata las mismas reglas sintácticas que los auxiliares típicos.

Basta intentar las transformaciones normales a la forma pasiva de (4.2) para confirmar la sospecha de que la estructura profunda de ese enunciado normativo no ha sido correctamente analizada en su génesis. En efecto, advertimos que de (4.2) podemos derivar varias oraciones pasivas correspondientes. Por ejemplo, las siguientes:

(7.4) El comprador debe (tiene el deber que el precio sea pagado (por el comprador).

(7.5) Es debido por el comprador que se (el comprador) pague el precio.

(7.6) Es debido por el comprador que el precio sea pagado (por el comprador).

Es decir: pareciera que la transformación de pasivo jugara tanto con respecto de la frase verbal, como con respecto del constituyente compuesto por la frase nominal y el auxiliar modal, y, por fin, que afectara tanto a la frase nominal como a la frase verbal y sus respectivos constituyentes. La segunda posibilidad, por ejemplo, exhibe una forma pasiva que afecta principalmente a la frase nominal y al auxiliar modal; en (7.6) la transformación pasiva pareciera operar por separado sobre la frase nominal y su verbo modal, y sobre la frase verbal y su objeto. Todo ello sugiere que la interpretación de 
(4.2) propuesta en el árbol generativo (7.2) es insuficiente, en cuanto en la estructura profunda de un enunciado normativo tan simple y típico podrían darse más constituyentes que habrian quedado eliminados por transformaciones no puestas suficientemente en claro.

8. Esta posibilidad es analíticamente más rica y permite una interpretación más adecuada de los enunciados normativos como (4.2), y, por tanto, de lo que Gioja denominaba "la forma de todo conocimiento normativo". La prueba consistente en someter (4.2) a otras transformaciones típicas, como la de nominalización, confirma que el análisis de la estructura profunda intentado en el parágrafo anterior es insuficiente. $Y$, por lo tanto, que también lo es la analogía adoptada, para el lenguaje natural, a partir de una regla de formación propuesta para los lenguajes artificiales de los sistemas deónticos. En suma: cuando nos referimos a la regla lingüística que permite pasar, por transformación sintáctica, de una oración meramente enunciativa a un enunciado normativo asociado, no se trataría de una función gramatical definitoria de un par ordenado $(p / O p)$, sino más bien de una función gramatical que asocia a un enunciado $p$, una clase de enunciados cuyos miembros están constituidos en cada caso por el primitivo enunciado $p$, más un enunciado $p_{1}$ que, en la estructura profunda, modaliza al primero. Este modelo analítico tiene la ventaja de que no requiere introducir, en la investigación del lenguaje natural, una categoría de constituyentes ad hoc, como específicos auxiliares modales idiosincráticos (el sollen kelseniano y sus oscuras características) o como operadores análogos a los operadores lógicos deónticos (analogía poco admisible dadas las diversas funciones cumplidas por los unos en lenguajes artificiales construidos, y por los otros en un lenguaje natural dado); basta en cambio manejarse con la regla de formación normal de enunciados en los lenguajes naturales, que suele escribirse ${ }^{13}$

$$
\mathrm{S} \rightarrow \mathrm{FN}+\mathrm{FV}(\mathrm{S})
$$

regla que ciertamente rige también la construcción de oraciones castellanas sintácticamente bien formadas. ${ }^{14}$ En un plano ingenuo, esta interpretación parte de la sencilla comprobación de que el verbo paradigmático, cuyo estudio parece de tanta importancia para la comprensión de todo enunciado normativo - el verbo sollen, devoir, deber, etcétera- es también un común verbo transitivo.

13 Cf. N. Chomsky, Syntactic Structures (1956), capitulos 5 y 7 , y especialmente Aspects of the Theory of Syntax ( 1965$)$, capitulo $1, \$ 2$ y 4 .

$14 \mathrm{La}$ fórmula (8.1) es una regla de reescritura: una oración (S) puede descomponerse en una frase nominal más una frase verbal, la cual puede contar además con un componente optativo formado por otra oración, elemento este último que asegura la recursividad de la fórmula. 
En consecuencia, propongo como estructura profunda de (4.2) la siguiente:

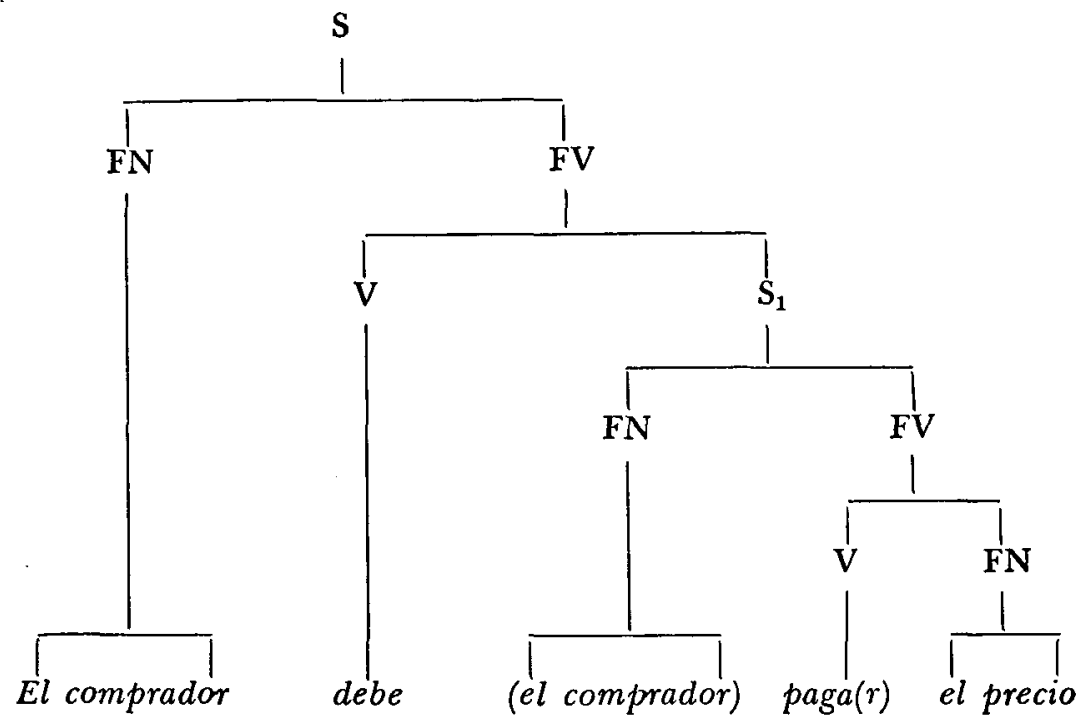

Conforme a una transformación corriente y bien conocida, la frase nominal de la oración subordinada $S_{1}$ es eliminada, por redundante, toda vez que es idéntica con la frase nominal de la oración principal $S$; el verbo principal, "debe", asume todos los rasgos morfológicos y sintácticos, mientras que el verbo de la oración subordinada, "paga", adopta la forma neutra del infinitivo, evitando así también una innecesaria redundancia en la aparición de rasgos verbales distintivos. La estructura de (4.2) esbozada en el árbol de (8.2) permite comprender la razón de que haya sido posible, en (7.4)-(7.6), formular varias oraciones pasivas derivadas, puesto que tanto cabe pasar a pasiva la oración principal $S$, como la subordinada $S_{1}$, así como también imponer esa transformación usual a ambas. Tenemos, por lo tanto:

(8.3) (Que) el comprador pague el precio es debido por el comprador;

(8.4) El comprador debe (tiene el deber) que el precio sea pagado (por él);

(8.5) Es debido (por el comprador) que el precio sea pagado (por el comprador).

Advertimos así que una de las formas corrientes de traducir, al lenguaje natural, el operador deónico $O$ (como cuando $O p$ es leído "es obligatorio que $p^{\text {") }}$ no constituye sino una de las posibles versiones pasivas de un enun- 
ciado normativo que, por serlo, incluye una oración subordinada en su seno (una embedded sentence, en la terminología de Chomsky). ${ }^{15}$

9. Tendríamos que, como resultado de la prueba a que hemos sometido a (4.2), sujetándola a la transformación de pasiva, hemos encontrado que la modalización deóntica - aquella estructura lógica inédita que, según Gioja, habría permitido a Kelsen encontrar la forma de todo conocimiento jurídico en ciertos juicios de deber ser- supone una estructura profunda en donde, por lo menos, aparece un enunciado imbricado y transformado, al punto de que en (4.2) difícilmente podría extraerse del análisis de la estructura superficial que estuviéramos ante una oración compuesta, de un enunciado molecular. Parece claro que, para este análisis, el verbo "deber" no es ni la versión lingüística de un operador modal monádico, ni un verbo auxiliar modal ordinario, ni una conectiva proposicional. Es, más bien, un verbo transitivo corriente, tal como aparece en la siguiente oración:

(9.1) El comprador debe el precio.

La relación lógica - y ya no puramente sintáctica o gramatical- entre $S$ y $S_{1}$ en (8.2) seguramente puede ser pensada de muy diversas maneras, según sea la formalización lógica a que se desee someter el enunciado, habida cuenta que toda formalización es relativa a los esquemas inferenciales a que se someta el enunciado formalizado. De ahí que no sólo no ténga mayor sentido buscar la supuesta forma lógica única y exclusiva de las normas jurídicas, puesto que los esquemas deductivos son infinitos, sino que tampoco cabe proponer un esquema único —el juicio de deber ser- como modelo exclusivo de los enunciados normativos en los lenguajes naturales. También las normas son formadas conforme a una regla como la de (8.1) que es recursiva.

Cabe pensar que el resultado del análisis efectuado -la simple norma formulada en (4.2) aparece como una compleja estructura que incluye oraciones subordinadas- permita aclarar algunos problemas formales del análisis lógico de las expresiones normativas, como ser los derivados de las negaciones internas y externas del modalizador, o los de la interpretación de la reiteración de los modalizadores.

Más importante, quizás, sean las perspectivas que se abren para la interpretación semántica de los enunciados normativos. Así, si la interpretación propuesta de (4.2) es correcta, parece claro que la oración subordinada interna $S_{1}$ tiene una referencia semántica suficientemente clara, como claro puede ser su eventual valor de verdad. Pero el enunciado molecular (4.2) es complejo si bien la referencia de $S_{1}$ es la misma que la que se atribuya a (4.1), la oración principal $S$ también puede ser vista como semánticamente significativa, ateniéndose al modelo simple ofrecido en el ejemplo de (9.1).

15 Cf. N. Chomsky, Aspects of the Theory of Syntax, cap. I, $\S 2$. El traductor castellano, C. P. Otero, traduce "oraciones (o construcciones) incrustadas". 
Posiblemente las transformaciones suscitadas por la constitución imbricada de (4.2) permitan comprender con mayor claridad la propuesta de Kelsen de derivar hacia las Rechtssätze, las proposiciones juridicas, la cuestión de la verificación de los enunciados jurídicos. ${ }^{16} \mathrm{Y}$ también la estructura profunda revelada en (8.2) permite comprender cómo la transformación lingüística que autoriza a formular una norma sin trazas de lexema o morfema normativo alguno - como nuestro ejemplo (2.3) - consiste en la supresión de una oración principal que puede ser sustituida por rasgos suprasegmentales (como cuando se la formula imperativamente), o por restricciones pragmáticas impuestas por el contexto de uso.

Universidad de Buenos Aires

ROBERTO JOSÉ VERNENGO

16 H. Kelsen, op. cit., $\S_{16}$, especialmente p. 76 ss. 